\title{
Audit of neonatal intensive care transport
}

\author{
A J Leslie, T J Stephenson
}

Although in utero transfer is the safest option, ${ }^{12}$ provided the mother's condition allows, many newborn infants are still transferred from their hospital of birth to another hospital to receive intensive care. There have been audits of the transfer of both adults ${ }^{34}$ and older children ${ }^{5}$ to intensive care units in the United Kingdom but there has been no published audit of the work of a neonatal 'flying squad'. Such audits have been carried out in the United States, ${ }^{6-8}$ but these data cannot be extrapolated to the United Kingdom because of the different organisation of perinatal care and the greater distances involved in the United States. This type of audit can provide important information for a unit wishing to start a transport team, for staff serving on the call-outs, for purchasers and providers negotiating contracts, and for regional authorities trying to rationalise local agreements in order to avoid expensive duplication of resources in response to market forces. We report an analysis of the work of our neonatal transport team in collecting sick infants over a recent 19 month period and discuss the clinical and cost implications.

\section{Methods}

PERSONNEL AND EQUIPMENT

The neonatal transport team consisted of at least one doctor and one nurse from the neonatal intensive care unit. The transport ambulance was provided by Nottinghamshire Ambulance Service. The equipment used was a transport incubator (Vickers 770, Vickers Medical), mounted on a customised trolley to fit a York 4 ambulance fitting, with an integral positive pressure ventilator. The ventilator could be adjusted to deliver flow rates up to $16 \mathrm{1} / \mathrm{min}$ and to ventilate at rates of up to $150 \mathrm{breaths} / \mathrm{min}$, inspiratory:expiratory ratio of $1: 1$ to $1: 50$, peak inspiratory pressures of up to $48 \mathrm{~cm} \mathrm{H}_{2} \mathrm{O}$. The system has a 'choke' on the expiratory limb to allow positive end expiratory pressure up to $15 \mathrm{~cm} \mathrm{H}_{2} \mathrm{O}$. The system includes a compressor that can be supplied from the electrical mains, the ambulance engine battery, or a portable 12 volt battery attached to the transport system. The compressed air could be mixed with oxygen from a portable cylinder or from a wall oxygen point, in the hospital or on the ambulance, to deliver a fractional inspired oxygen $\left(\mathrm{FiO}_{2}\right)$ from $0 \cdot 21-1 \cdot 0$. Gas to the ventilator could not be heated or humidified during the period which we describe. The ventilator circuit included a disconnection alarm. The $\mathrm{FIO}_{2}$ was continuously monitored with a portable oxygen analyser (Model 273, Vickers Medical). It was possible to monitor each infant with the following equipment: a continuous electrocardiogram (ECG) (Micromon 7142, Kontron Instruments Ltd) with an oscillographic display and digital heart rate; a pulse oximeter (Minolta Pulseox-7, DeVilbiss Healthcare UK Ltd) with a signal strength display and continuous digital display of heart rate and saturation; a transcutaneous oxygen electrode (Cutan 820, Kontron Instruments Ltd), heated to $44^{\circ} \mathrm{C}$, with digital display of transcutaneous oxygen tension; axillary and peripheral thermocouples (Model 272 Vickers Medical) with continuous digital displays of the absolute temperatures to one decimal place and the derived core-peripheral difference. In infants with umbilical arterial catheters including an indwelling oxygen electrode ('Searle' Catheter, Biomedical Sensors), arterial oxygen tension $\left(\mathrm{PaO}_{2}\right)$ was monitored continuously using a Neocath 1000 Monitor (also Biomedical Sensors) but this was not standard equipment. There were no facilities for invasive or oscillometric monitoring of arterial blood pressure during the period of the study. Up to six intravenous or intra-arterial infusions could be given using $50 \mathrm{ml}$ syringe drivers (MS2000, Graseby Medical). All monitoring equipment and pumps had individual internal battery supplies for use in transit.

\section{STUDY PROTOCOL}

Every infant transferred into our unit by our transport team was included in the study, for the period August 1991 to February 1993 (19 months; $n=73$ ). Those not included were babies collected by a nurse alone (that is, well babies transferred back to our unit after intensive care elsewhere; $n=7$ ); babies brought by another hospital's transport team $(n=38)$; and babies transferred out for care elsewhere (to a cardiac centre or because our unit was full; $\mathrm{n}=31$ ).

All data were collected prospectively by the doctor and the nurse comprising the transport team on that occasion. The following were recorded: demographic details of the baby and details of the infant's problems, referral centre, time referral accepted, time ambulance left our unit, time of arrival of the team at the referring neonatal unit, time of departure from the 
Table 1 Scoring system to evaluate a transported infant's condition (after Hermansen et $\mathrm{al}^{7}$ )

\begin{tabular}{|c|c|c|c|}
\hline & \multicolumn{3}{|l|}{ Score } \\
\hline & 0 & 1 & 2 \\
\hline Blood glucose $(\mathrm{mmol} / \mathrm{l})^{\star}$ & $<1 \cdot 4$ & $\begin{array}{c}1 \cdot 4-2 \cdot 2 \\
\text { or }>9 \cdot 7\end{array}$ & $2 \cdot 3-9 \cdot 7$ \\
\hline $\begin{array}{l}\text { Systolic blood pressure }(\mathrm{mm} \mathrm{Hg}) \\
\mathrm{pH}\end{array}$ & $\begin{array}{l}<30 \\
<7 \cdot 2 \\
\quad \text { or }>7 \cdot 5\end{array}$ & $\begin{array}{l}30-39 \\
7 \cdot 2-7 \cdot 29 \\
\quad \text { or } 7 \cdot 46-7 \cdot 50\end{array}$ & $\begin{array}{l}>39 \\
\quad 7 \cdot 30-7 \cdot 45\end{array}$ \\
\hline $\mathrm{PaO}_{2}(\mathrm{kPa})$ & $<5.3$ & $\begin{array}{l}5 \cdot 3-6 \cdot 5 \\
\text { or }>13 \cdot 3\end{array}$ & $6 \cdot 6-13 \cdot 3$ \\
\hline Core temperature $\left({ }^{\circ} \mathrm{C}\right)$ & $\begin{array}{l}<36 \cdot 1 \\
\quad \text { or }>37.7\end{array}$ & $\begin{array}{l}36 \cdot 1-36 \cdot 5 \\
\quad \text { or } 37 \cdot 3-37 \cdot 6\end{array}$ & $36 \cdot 6-37 \cdot 2$ \\
\hline
\end{tabular}

^Measured by reagent strips (BM Stix).

referring hospital, time of arrival on return to our unit, equipment used during transfer, any equipment problems, maximum ventilation requirements during transfer, number and type of infusions, any medical problems with the baby during transfer.

A 'transport score' was calculated contemporaneously by the transport team as shown in table $1 .{ }^{7}$ The first score was obtained on arrival at the referring unit and the final score as soon as possible after transfer to our unit. No extra investigations beyond those clinically indicated were done in order to complete the scoring system.

\section{STATISTICAL METHODS}

For demographic data, medians with interquartiles are given in addition to the range. Measurements of central tendency and dispersion are more useful to those primarily interested in costs; the maximum and minimum variables encountered are important for the clinical and technical staff who must provide skills, facilities, and equipment that will cover even the most extreme possibilities. Paired comparisons were made using Wilcoxon signed rank test and correlation between variables was estimated using Spearman's rank correlation.

\section{Results}

(1) DETAILS OF INFANTS TRANSFERRED

Data were collected on 73 call-outs. No infant was transferred more than once. The median birth weight was $1590 \mathrm{~g}$ (minimum 500, 25th centile 1060,75 th centile 2420 , maximum 3920 ) and the median gestation was 31 weeks (minimum 23, 25th centile 28,75 th centile 36 , maximum 42). Seventy five per cent were transported on the first day of life, and $50 \%$ of the total at 12 hours of age or less. The

Table 2 Principal problem for which transfer was requested

\begin{tabular}{ll}
\hline $\begin{array}{l}\text { Respiratory distress syndrome: } \\
48\end{array}$ & $\begin{array}{l}\text { Asphyxia: } 5 \\
\text { Gastroschisis: } 2\end{array}$ \\
Necrotising enterocolitis: 3 & Pneumonia: 2 \\
Exomphalos: 2 & Neurosurgical opinion: 2 \\
Bowel obstruction: 2 & Diaphragmatic hernia: 2 \\
Sepsis: 2 & Duodenal atresia: 1 \\
Infant of diabetic mother: 1 & Pneumothoraces: 1 \\
Failure to thrive: 1 & Treacher Collins syndrome: 1 \\
Achondroplasia: 1 & Meconium aspiration syn- \\
Hydrops fetalis: 1 & drome: 1 \\
Persistent ductus arteriosus: 1 & Persistent pulmonary hyper- \\
Tracheo-oesophageal fistula: 1 & tension: 1 \\
Intrauterine growth retarda- & Apnoeas: 1 \\
tion: 1 & \\
\hline
\end{tabular}

remainder were transported at varying postnatal ages, up to 504 hours. These older infants were usually referred for surgical management.

Forty eight infants were transferred because intensive care or surgical facilities were not available in the hospital of birth; 24 infants were transferred because the intensive care facilities in their hospital of birth were full; one infant was born at home. The principal clinical problems at the time of referral are listed in table 2 . Of these 73 babies, 68 were identified as having a major medical problem, and 15 had significant surgical problems, some infants having both.

The 73 transfers came from 16 different hospitals (and one home delivery), both inside and outside the Trent region, the maximum distance being 80 miles from Nottingham. Most of these centres are within health authorities with which the Nottingham City Hospital trust has a block contract (largely those with which there were historical links, and for which the City Hospital had responsibility as a referral centre during the period before neonatal intensive care was deregionalised), whereas others represent hospitals within areas paying an extracontractual referral fee for each case (fig 1). Ultimate responsibility for payment depends upon the home postcode of the patient.

\section{(2) TRANSPORT TIMES}

(A) Response time

This is derived from the time of telephone acceptance of the transfer until the ambulance was recorded as being mobile. The median response time was 45 minutes $(n=72)$ (minimum 10,25 th centile 35,75 th centile 65 , maximum 200). Important limitations on response times included arranging cross cover for the attending medical and nursing staff, and for all out of hours transfers, arranging for the on-call doctor to come in from home. This lead to a disparity of response time between calls accepted in and out of 'office hours'. The median response time Monday to Friday 8 am to $6 \mathrm{pm}$ was 40 minutes $(\mathrm{n}=20)$, and the median time outside these hours was 50 minutes $(n=52)$. Although these medians are similar, all the response times over 105 minutes occurred out of hours.

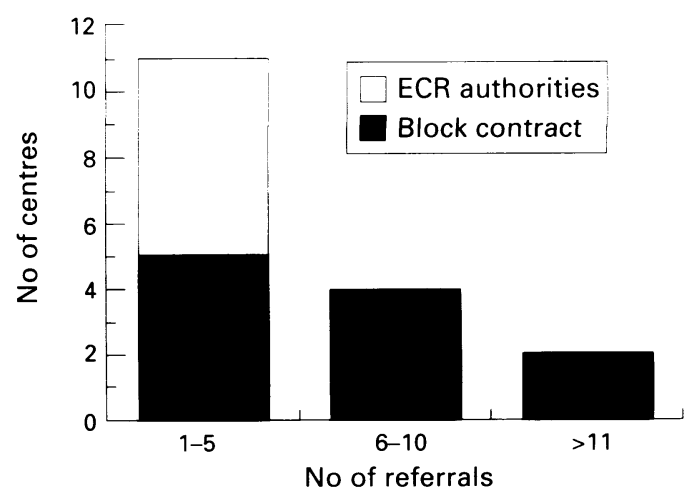

Figure 1 Figure shows that most centres refer only a small number of infants each year. Extracontractual referral (ECR) authorities are those with whom Nottingham does not have a block contract. 


\section{(B) Stabilising time}

This is derived from time of arrival at the referring centre to the time of departure from the referring centre. Activities performed within this period included procedures to the baby, the optimising of management, obtaining informed consent if surgery was indicated, attending to note and information gathering, and seeing parents to explain about the baby's condition, the need for transfer, and any other matters they wished to discuss. One or other parent was seen before departure in 70 cases; on another two occasions the parents were already in transit to Nottingham.

The median time spent stabilising was 75 minutes $(n=71)$ (minimum 3, 25th centile 60 , 75th centile 95 , maximum 180 ). The minimum was for the only infant in the cohort collected following an unplanned home delivery.

\section{(C) Total transfer time}

This was counted from the time the ambulance was deemed mobile until the time of arrival back at our unit. Over this 19 month period the team was out for a total of 233.75 hours. The median time per call was $3 \cdot 1$ hours $(n=72)$ (minimum $0 \cdot 15,25$ th centile $2 \cdot 4,75$ th centile 4, maximum 5·75).

\section{(3) EQUIPMENT USE}

All babies except one had their heart rate continuously monitored on the ECG machine. All except two babies had continuous measurement of central temperature. Intravenous or intra-arterial infusions were given from a $50 \mathrm{ml}$ syringe via a syringe driver. All except two babies required at least one intravenous infusion, and the maximum needed was four (median 2). Thirty three babies $(45.2 \%)$ had a peripheral or umbilical arterial catheter in place for the journey. The commonest equipment problems encountered during transfer were interference on the ECG trace and intermittent loss of signal from the pulse oximeter.

\section{(4) RESPIRATORY SUPPORT}

In each case, the maximum level of support required during the journey was recorded.

Respiratory support was needed by 71 babies $(97 \cdot 3 \%)$. Eight babies $(11 \%)$ were managed with increased ambient oxygen alone. They were given extra oxygen in a range from 30 to $70 \%$ (mean $45 \cdot 2 \%$ ). Two were managed with nasal continuous positive airway pressure, $4-5 \mathrm{~cm} \mathrm{H}_{2} \mathrm{O}$, and the remaining 61 $(83 \%)$ received intermittent positive pressure ventilation. The median peak pressure was 20 $\mathrm{cm} \mathrm{H}_{2} \mathrm{O}$ (maximum 36 ), the median rate was 60 breaths per minute (maximum 84), and the median inspired oxygen was $65 \%$ (maximum 100 ). The adequacy of oxygen delivery was assessed using a pulse oximeter in 66 babies $(90.4 \%)$, a transcutaneous oxygen electrode in 14 babies $(19 \cdot 2 \%)$, and a Neocath umbilical catheter in five $(6 \cdot 8 \%)$.
There were three incidences of serious complications of ventilation: two tube blockages, which were replaced successfully (in one case further resuscitation was needed) and one accidental extubation. This tube was left out and the baby managed in extra ambient oxygen for the remainder of the journey.

\section{(5) costs}

A charge of $£ 1680$ was made per transfer, either per case for extracontractual referrals or included in block contracts. This sum, calculated in 1992, was derived from estimated nursing and medical staff costs, with a further component included for equipment depreciation, and divided by the total number of acute transfers.

This may not accurately reflect the true cost of transport, as some major items, for example ambulance charges, were not available for inclusion at the time that the costs were calculated. Moreover, the medical staff costs included only 'on-call' payments for transfers out of hours.

\section{(6) OUTCOME}

No baby died in transit. Seventeen patients died $(24 \%)$ subsequently, before discharge from our unit, of whom five died within 24 hours of admission. These mortality data emphasise that infants who are transferred are a highly selected group, many of whom are in a very unstable condition. The median duration of stay was 11 days $(n=72)$ (minimum 1,25 th centile 5,75 th centile 21 , maximum 147 ). These data do not include one infant with a congenital diaphragmatic hernia, pulmonary hypoplasia, and short gut syndrome who remains on the neonatal unit six months after referral.

\section{(7) RESULTS OF TRANSPORT SCORES}

\section{(A) Paired comparisons}

For each individual variable (blood glucose, blood pressure, $\mathrm{pH}, \mathrm{PaO}_{2}$, and temperature) scores were obtained on arrival of the transport team and again after the transport was completed. Scores were examined to determine whether they improved, worsened or were unchanged, and these data compared using the Wilcoxon signed rank test, as shown in table 3 .

\section{(B) How were points lost?}

Each variable can score 0,1 , or 2 points. Figure 2 shows the number of points lost for each variable, as a percentage of the total possible score. This is shown for the two occasions when this was assessed: when the transport team arrived and after completion of transport.

(C) Use of scores for prediction

Hermansen et al related level of score to outcome. ${ }^{7}$ They used a score of 8 as a predictive 
Table 3 Paired comparisons on the changes in score, allowing for tied ranks (Wilcoxon signed rank test), before and after transfer, of blood pressure, blood gases, glucose, and temperature

\begin{tabular}{lllcll}
\hline & $\begin{array}{l}\text { Score } \\
\text { increased }\end{array}$ & $\begin{array}{l}\text { Score } \\
\text { unchanged }\end{array}$ & $\begin{array}{l}\text { Score } \\
\text { fell }\end{array}$ & No & p Value \\
\hline Blood glucose & 14 & 44 & 6 & 64 & $0 \cdot 049^{\star}$ \\
Systolic blood pressure & 9 & 48 & 4 & 61 & $0 \cdot 21$ \\
$\mathrm{pH}$ & 17 & 27 & 21 & 65 & $0 \cdot 81$ \\
$\mathrm{PaO}_{2}$ & 16 & 44 & 6 & 66 & $0 \cdot 019^{\star}$ \\
$\mathrm{Temperature}^{\text {Temp }}$ & 21 & 36 & 9 & 66 & $0 \cdot 021^{\star}$ \\
Total score & 29 & 21 & 14 & 64 & $0 \cdot 003^{\star}$ \\
\hline
\end{tabular}

An asterisk denotes significance at the $\mathrm{p}<0.05$ level.

level, and showed poor outcome below that level and good outcome at a score of 8 or over. Our data for the same critical level of score, with outcome being defined by survival to discharge from the neonatal unit, are shown in table 4.

Data from score 1 (prestabilisation) - These data yield a sensitivity of $66 \%$ (per cent dying whose score was under 8 ), specificity $69 \%$ (per cent of survivors whose score was over 8), positive predictive value $39 \%$ (per cent of all those with a score under 8 who died), and negative predictive value $88 \%$ (per cent of all those with a score over 8 who lived).

Data from scores 1 and 2 - A score of less than 8 in either total (before or after transfer) is used as the predictive measure. These data yield a sensitivity of $80 \%$, specificity $63 \%$, positive predictive value $40 \%$, and negative predictive value $91 \%$.

\section{Discussion}

Within the Trent region, for the period 1 April 1990 to the end of March 1992, there were about 950 babies per year born at less than 32 weeks' gestation and/or less than $1500 \mathrm{~g}$ birth weight (S Bohin and D Field, personal communication). The total number of urgent flying squad transfers into the five referral centres (City Hospital Nottingham, University Hospital Nottingham, Leicester Royal Infirmary, Jessop Hospital for Women Sheffield, and Derby City Hospital) was 314 for the first year of that period and 235 for the second year. Taking the 73 babies that we report here and adding the 38 babies brought

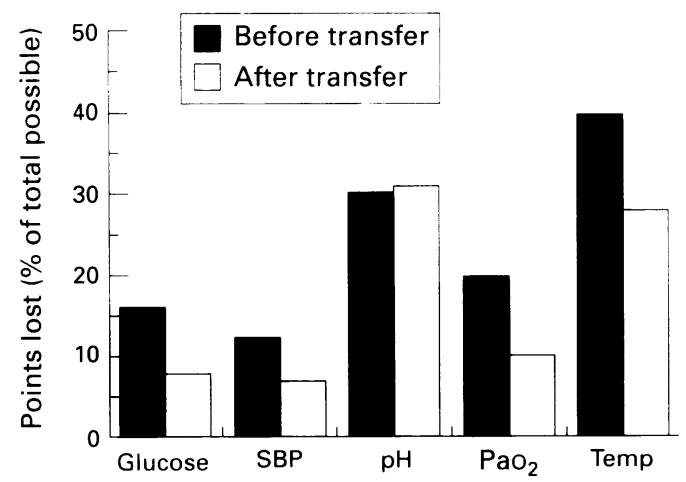

Figure 2 Percentage of total possible points lost for each variable at two points in time: before intervention by the transport team at the referring hospital, and after completion of transport. ( $S B P=$ systolic blood pressure; Temp = temperature.)
Table 4 Relationship between transport scores and a fatal outcome for those infants for whom complete score data was available (see text for details of scoring system). Score 1 was recorded at the time of arrival of the flying squad and score 2 at completion of the transfer

\begin{tabular}{|c|c|c|}
\hline & $\begin{array}{l}\text { Survived to } \\
\text { discharge }\end{array}$ & Died \\
\hline Score $1<8$ & 16 & 10 \\
\hline Score $1 \geqslant 8$ & 35 & 5 \\
\hline \multirow{2}{*}{\multicolumn{3}{|c|}{ One (that is, score 1 or 2 ) or both (that }} \\
\hline & 18 & 12 \\
\hline Both totals $\geqslant 8$ & 31 & 3 \\
\hline
\end{tabular}

by another hospital's transport team, this gives a figure of five or six babies per month arriving by urgent flying squad at the City Hospital Nottingham. The great variety of the work involved can be seen from the list of diagnoses in table 2 . Although $65 \%$ of babies had respiratory distress syndrome due to preterm delivery as the major reason for transfer, no other single problem occurred on more than five occasions during this period and in all there were 23 different diagnoses given as the major reason for referral. Fifteen of the referrals were principally for surgery or surgical opinion, whereas the remainder were for neonatal intensive care for paediatric medical problems.

Referrals came from 16 different centres and it is important that both the ambulance centre and the flying squad have detailed information about the best route to take at different times of day to get to these centres, current delays or roadworks, a detailed map of the hospital showing the closest point to the neonatal intensive care unit at which the ambulance can park and how to get from there to the neonatal intensive care unit. The referral centre must also have some idea of the expertise and limitations of all 16 centres as this may dictate which staff should go on the transfer, which equipment should be taken, and what length of delay is reasonable. It is clear from this audit that 'flying squad' is something of a misnomer; a median response time of 45 minutes emphasises that we are providing a transport service but not a ' 999 ' response. The minimum response time in the study was 10 minutes (for the home delivery) but this is rarely feasible, particularly out of hours, and this has relevance to current plans to increase the proportion of home deliveries in the United Kingdom.

This audit showed that a quarter of infants transferred in stay for longer than three weeks. Hence, any unit undertaking neonatal transfers must be prepared to provide the full panoply of social and psychological support, long term, for parents who may be a long way from their home and families. It is also clear that any centre accepting flying squad referrals may have their own capacity for providing intensive care to locally born infants limited for a long period and cost should reflect this. In the period $1990-2$ in the Trent region, 30-40 babies per year were identified who had to receive intensive care in a referral centre which was not their first 'port of call' geographically (S Bohin and D Field, personal communication). Our data would suggest that part of this 


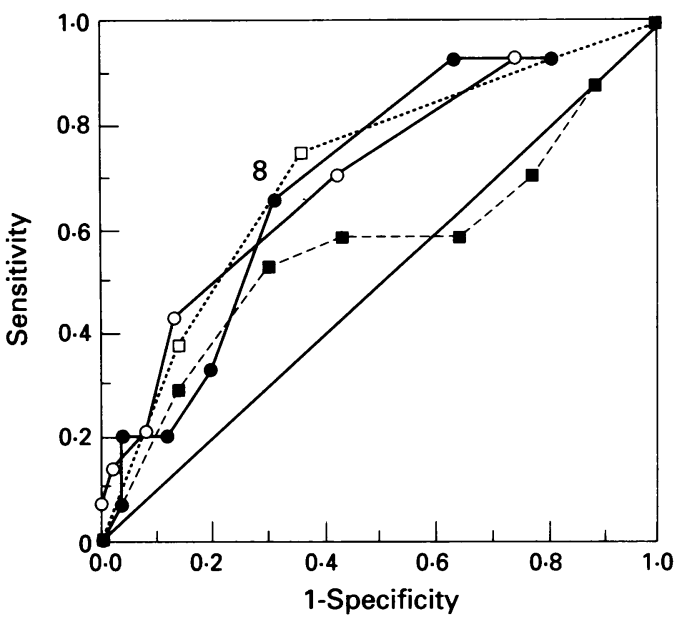

- Before transfer total score
- - After transfer total score
$-\square-$ After transfer temperature score
- Birth weight
- Toss of a coin

Figure 3 ROC curves (see text for explanation) for prediction of hospital mortality by transport score, after transfer temperature, and birth weight. The solid circle marked 8 shows the specificity and sensitivity when a before transfer score of less than 8 is used to predict outcome.

logistical inefficiency may arise due to long stay flying squad referrals.

Most of the infants transferred were extremely small, preterm, and less than a day old. These babies present major medical problems to the transport staff as they are a highly selected group of the most vulnerable of the preterm population. It also presents a considerable challenge to the equipment used which, on the whole, has been designed for a static base within a well appointed neonatal intensive care unit and not for usage within a confined space at high speed and with multiple sources of interference. Most babies were monitored using pulse oximetry and although signal loss is notoriously frequent with this modality, we found that if the probe was well secured the motion artefact problems could be minimised. A well applied transcutaneous oxygen monitor gives more continuous data, although of course has a slower time response after deterioration in the infant. Best oxygen monitoring data is obtained from an umbilical arterial catheter with an indwelling oxygen electrode and continuous readout. This carries a small risk of accidental removal during the journey and attendant blood loss.

The vast majority of babies were intubated during the transfer. Some of these babies were not intubated at the time when the transport team arrived but were intubated before transport, either because their condition dictated this or it was felt likely that their condition might deteriorate during transport. Intubation during transport should be avoided if at all possible. No pneumathoraces were detected during these 73 transfers; insertion of a chest drain during flying squad transfer is probably the most demanding of all procedures. Oral endotracheal tubes were used in all intubated transfers and, generally, rates of $50-80$ per minute were used with diamorphine sedation. The flying squad were reluctant to use paralysing agents before transfer because of the risk of accidental extubation.

\section{TRANSPORT SCORES}

The transport scores, as described in this paper, were first devised by Hermansen et al. ${ }^{7}$ The scores for each parameter are shown in table 3. There are not scores for all 73 babies because some infants did not have an arterial line and did not justify a capillary blood gas (for example, a stable term infant with duodenal atresia). One advantage of the scoring system is that it looks at five variables which may all be affected by transport team interventions, rather than concentrating on one variable alone, and the scoring requires no extra work by the transport team as all these data would be routinely collected as they are clinically relevant. Figure 2 shows that most points were lost by both the referral centre and the transport team for temperature and $\mathrm{pH}$. The degree of acidosis may be a reflection of the illness of the infant. Despite changes in ventilator settings, correction of hypovolaemia and administration of bicarbonate, many infants will remain acidotic. In contrast, however, the maintenance of a normal core temperature is one of the essentials of basic neonatal care. The observation that many points lost by the transfer team were for temperature is a cause for concern. The equipment used by the transport team was not all designed specifically for this purpose and much was customised from equipment routinely used in the neonatal intensive care unit. In order to maintain body temperature throughout the transfer, particularly as frequent access to the infant may be required, new incubator designs may be necessary.

An alternative way of looking at predictive accuracy of a scoring system is to plot the receiver operating characteristic (ROC) curve. These are shown for the total before transfer and after transfer scores in fig 3. The curves are obtained by taking each possible value for the score and plotting 1-specificity versus the sensitivity when that score is used as the cut off to predict a fatal outcome. For example, if a before transfer score of less than 8 is used to predict death (and thus $\geqslant 8$ predicts survival), the sensitivity if $66 \%$ and the specificity $69 \%$ giving the point marked as 8 on the graph. In general, high scores give high sensitivity but poor specificity at predicting death (top right corner of the ROC plot) whereas low scores give high specificity but poor sensitivity at predicting death (bottom left corner of the ROC plot). The best score to use as a cut off for prediction, giving the best combination of high sensitivity and specificity, is the point on the curve nearest the top left corner. The area under the curve gives an overall measure of the usefulness of the score in predicting the outcome, irrespective of the cut off chosen.

Both the before transfer and after transfer scores are better than birth weight alone at predicting outcome but there is not much to 
choose between the two scores and neither is a very powerful predictor. More interestingly, the after transfer temperature score alone is as useful as either total score in predicting outcome. We were concerned that low core temperature may simply have been acting as a proxy for immaturity but there was no significant correlation between after transfer temperature and either birth weight or gestation (Spearman rank correlation) and, moreover, the area under the ROC curve for temperature is greater than for birth weight alone. Hence, after transfer temperature does seem to be an independent predictor of outcome, although this does not allow us to distinguish whether this is causal or association. In contrast, before transfer temperature score correlated with birth weight $(p=0.02)$ and before transfer $\mathrm{pH}$ $(p=0.005)$ suggesting that hypothermia in the referring hospital was simply a marker for the smallest, sickest babies. Taking these findings together with table 3 , it appears that the transport team were generally effective in maintaining or improving core temperature (thus abolishing the relationship between temperature, size, and $\mathrm{pH}$ which existed in the referring centre). However, of the 17 infants who subsequently died, only five had a normal before transfer temperature and this proportion was unchanged after transfers, explaining why this variable is predictive of outcome.

\section{CONCLUSIONS}

We conclude that all units undertaking flying squad transfers should perform regular audit of their data to look for ways in which they can improve the quality and speed of transfer. The use of a transport scoring system allows both referral units and transport teams to concentrate on those areas where points are being lost unnecessarily. Just as Apgar scores have limitations but provide a shorthand that is widely understood, transport scores provide a rapid objective and statistically useful basis for comparing the condition of transported infants. If the score is above 8 when the transport team arrives and remains above 8 when the transport team returns to the regional unit, it would seem that the outlook for survival is reasonably good. This audit has led to several innovations in our practice, including a formal sheet of questions to ask at the time of referral that may provide a more complete picture of the baby's condition, and for advice on management before arrival of the team to be given. A portable video unit is taken out on transports to improve the quality of the information that parents receive.

Our data would suggest that the widespread belief that infants invariably deteriorate during transfer is not true and this is a tribute to the skills of the transfer team given the often very unstable condition of these babies.

We are very grateful to Professor M J R Healy for statistical advice and to all the members of the Nottingham Neonatal Transport Service for help in collecting these data.

1 Kollee LAA, Verloove-Vanhorick PP, Verwey RA, Brand R, Ruys JH. Maternal and neonatal transport: results of a national collaborative survey of preterm and very low birth weight infants in the Netherlands. Obstet Gynecol 1988; 72: 729-32.

2 Lubchenco LO, Butterfield LJ, Delaney-Black V, Goldson V, Koops BL, Lazotte DC. Outcome of very-low-birth-weight infants: does antepartum versus neonatal referral have a better impact on mortality, morbidity, or long-term outbetter impact on mortality, morbidity, or long-t
come? Am $\mathcal{7}$ Obstet Gynecol 1989; 160: 539-45

3 Reeve WG, Runcie CJ, Reidy J, Wallace PGM. Current practice in transferring critically-ill patients among hospitals in the west of Scotland. BMF 1990; 300: 85-7.

4 Kee SS, Ramage CMH, Mendel P, Bristow ASE. Interhospital transfers by helicopter: the first 50 patients of the Careflight project. F $R$ Soc Med 1992; 85: 29-31.

5 Robb HM, Hallworth D, Skeoch CH, Levy C. An audit of a paediatric intensive care transfer unit. Examining the workload and practice of the medical team. British fournal of Intensive Care 1992; Nov-Dec: 371-9.

6 Ferrara A, Atakent Y. Neonatal stabilization score. A quantitative method of auditing medical care in transported tative method of auditing medical care in transported 1986; 24: 179-87

7 Hermansen MC, Hasan S, Hoppin J, Cunningham MD. A validation of a scoring system to evaluate the condition of transported very-low-birth-weight neonates. Am $\mathcal{f}$ Perinatol 1988; 5: 74-8.

8 Zwischenberger JB, Keeney S, Raymond G, Hanson M, Cox CS. Neonatal transport in Texas. Tex Med 1992; 88:
66-9. 